\title{
Characterization of the Gene Encoding Radish (Raphanus sativus L.) PG-inhibiting Protein
}

\author{
Byung-Ho Hwang ${ }^{1,2 \dagger}$, Hun Kim ${ }^{1 \dagger}$, Sooyeon Lim $^{1}$, NaRae Han ${ }^{1}$, and Jongkee Kim ${ }^{1 *}$ \\ ${ }^{I}$ Department of Integrative Plant Science, Chung-Ang University, Anseong 456-756, Korea \\ ${ }^{2}$ Institute of Molecular Plant Sciences, University of Edinburgh, King's Buildings, Mayfield Road, Edinburgh EH9 $3 J H$, UK
}

\begin{abstract}
A radish (Raphanus sativus L.) polygalacturonase-inhibiting protein (PGIP) gene was cloned and compared to the PGIP gene (BrPGIP2) from Chinese cabbage (Brassica rapa ssp. pekinensis) in order to gain more information on controlling a disease and improving produce quality. To clone the radish PGIP gene, primers were designed based on conserved sequences of two PGIP genes (BnPGIP1 and BnPGIP2) from rape (B. napus L. ssp. oleifera), Chinese cabbage and Arabidopsis thaliana. PCR cloning was performed with cDNA from the stigma of radish 'Daejinyeoreum' as a template to confirm DNA fragments which were about 600 base pair in size. Sequence analysis revealed $84.1 \%$ homology with $B r P G I P 2$ and $70.1 \%$ with BnPGIP1. DNA walking was conducted to confirm the open reading frame of $972 \mathrm{bp}$, and the gene was named RsPGIP1. RsPGIP1 consisting with 323 amino acids (aa) has a high leucine content (54/323) and contains 10 leucine-rich repeat domains, as do most BrPGIPs of Chinese cabbage. The gene expression of RsPGIP1 was induced by abiotic stresses and methyl jasmonate. It showed enrichment in the stigma and the primary root than a leaf. Cloning $R s P G I P 1$ will aid to further apply practices on postharvest quality maintenance and disease control of the root.
\end{abstract}

Additional key words: PGIP, polygalacturonase, postharvest disease, produce quality, radish

\section{Introduction}

Radish roots are one of the most popular vegetables in Korea, where people consume in a number of different food recipes, including a main ingredient of 'Kimchi'. Radish roots produced in spring are less susceptible to low temperature, whereas fall season types are very sensitive.

It is widely accepted that the firmness of radish root is one of the most important quality criterion that is used to processing 'Radish Kimchi' as well as other food recipes made of radish root (Cheigh and Park, 1994). Several studies suggested that pectins in cell walls play an important role in maintaining tissue firmness during processing or cooking of the root (Fuchigami, 1987; York et al., 1985).

The plant cell wall is an important barrier to pathogen invasion. Most plant pathogens secrete a cocktail of cell wall degrading enzymes capable of depolymerizing the polysaccharides in the plant cell wall. In response, plants have evolved a diverse battery of defense responses including proteinaceous inhibitors of pectin degrading enzymes such as PG (PGIP, PG inhibiting protein), pectinmethyl esterase (PMEI, PME inhibitor) and pectin lyase (PNLIP, PNL inhibitor protein), as well as hemicellulose degrading enzymes such as endoxylanase (XI, xylanase inhibitor), xylanase (XIP, X inhibitor protein) and xyloglucan endoglucanase (XEGIP, XEG inhibiting protein) (Juge et al., 2006). The majority of plant pathogenic fungi and bacteria secrete PG, which can degrade cell wall pectic components consisting of a rhamnogalacturonan main chain, and this seems to be involved in development of disease symptoms (Lebeda et al., 2001).

PGIPs that are present in plant cell wall have a leucinerich repeat (LRR) domain, one of the features of plant resistance proteins, and directly reduce the aggressiveness of fungal PG (Liang et al., 2005). The existence of PGIPs has been confirmed in leek and onion, which are monocots, and also in a number of dicotyledons including soybean,

\footnotetext{
*Corresponding author: jkkim@cau.ac.kr

${ }^{\dagger}$ These authors are contributed equally to this work.

※ Received 15 November 2012; Revised 24 December 2012; Accepted 29 December 2012. This work was supported by the Young Investigator Research Program of Chung-Ang University 2008 year, the GRRC program of Gyeonggi province [GRRC-CAU-2012-A01, Development of Baemoochae kimchi and postharvest technology] and the Korea Research Foundation Grant (KRF-2007-357-F00006) funded by the Korean Government (MOEHRD), Korea. The authors thank B. Han (Yeungnam University, Gyeongsan) for critical reading of the manuscript.
} 
apple, pear, orange, and kiwifruit. A variety of PGIP genes have been cloned from 103 plant species (De Lorenzo et al., 2001; NCBI gene bank). Recently, PGIP genes in rape, rice, strawberries and Chinese cabbage have been identified (Ahsan et al., 2005; Mehli et al., 2004). Most PGIP genes are composed of multi-gene families clustered in a particular chromosomal region. In the case of the two Arabidopsis thaliana PGIPs, they are located tandemly on chromosome 5 with a distance of 507 bp (Ferrari et al., 2003).

Numerous studies on PGIPs have investigated their expression in relation to invasion of fungal pathogens, the response to treatment with elicitors such as salicylic acid (SA), jasmonic acid (JA), and oligogalacturonides (OGs), and expression pattern after abiotic stress (De Lorenzo et al., 2001). In addition, the expression pattern of PGIP during developmental processes such as rice floral maturation and organ number has been reported (Jang et al., 2003). The expression of PGIPs of Brassica napus and Arabidopsis is regulated by some abiotic stresses (Ferrari et al., 2003).

The effect of apple PGIP was tested on a well characterized cell wall destructing enzyme, PG from a postharvest soft rot pathogen, Colletotrichum acutatum (Gregori et al., 2008). Recently, the genes encoding PGIPs were cloned and characterized in Chinese cabbage which is a main crop belonging to Brassicaceae family same as radish (Hwang et al., 2010).

Bacterial soft rot in radish root, caused by Pectobacterium carotovorum ssp. carotovorum $(P c c)$ is a soil epidemic disease. $P c c$ is a facultatively gram-negative bacterium and attacks plant tissue through the secretion of a cocktail of cell wall degrading enzymes mainly including PG during high temperature and high humidity like rainy spell in summer. Therefore, the study on inhibiting PG activity of $P c c$ is necessary.

In this paper, we cloned cDNA using RT-PCR after confirming the genomic sequence of RsPGIP gene from radish through PCR cloning and DNA walking, and investigated RsPGIP expression pattern in relation to environment stress and signaling molecules to find a useful way of applications for managing radish quality and diseases in postharvest.

\section{Materials and Methods}

\section{Plant Material and Sample Preparation}

Raphanus sativus L. 'Daejinyeorum' (Monsanto-Korea, Seoul, Korea) radish seed was immersed in water in dark conditions at $28^{\circ} \mathrm{C}$ for $36 \mathrm{~h}$, sowed in sterilized soil, and cultivated in a greenhouse for 4 weeks with a day temperature of 18 to $20^{\circ} \mathrm{C}$ and a night temperature 10 to $12^{\circ} \mathrm{C}$. For preparation of genomic DNA, 4-weeks-old radish leaves were extracted and stored at $-70^{\circ} \mathrm{C}$ after being frozen in liquid $\mathrm{N}_{2}$. We attempted to clone the PGIP gene from radish stigma through cDNA library screening based on the method used for the isolation of Chinese cabbage PGIP from stigma (Hwang et al., 2010). Ten young plants were placed at $4^{\circ} \mathrm{C}$ with $16 \mathrm{~h}$ day and $8 \mathrm{~h}$ night for 30 days to induce flower. After vernalization, plants were transplanted into $5 \mathrm{~L}$ pots and cultivated for 6 weeks in the greenhouse. When plants were in full bloom, stigmas were excised with a scalpel, immediately frozen in liquid $\mathrm{N}_{2}$, and stored at $-70^{\circ} \mathrm{C}$ for total RNA extraction.

For analysis of the expression patterns of RsPGIPl in various plant tissues, the 4 -week-old radish plants were placed at $4^{\circ} \mathrm{C}$ for 30 days under $16 \mathrm{~h}$ light conditions to induce flowering and then grown in the greenhouse until flowering. Stigma, leaf blade, and primary root tissues from flowering plants were harvested, frozen in liquid N2, and stored at $-70^{\circ} \mathrm{C}$ for later use.

To investigate the expression pattern after treatments with low temperature, salt stress, mechanical wounding, and defense response activators of $R s P G I P 1$, radish seeds were germinated at $28^{\circ} \mathrm{C}$ for $36 \mathrm{~h}$, and seedlings were grown in the greenhouse for 4 weeks. After selecting young uniform plants, they were conditioned for 5 days at $23^{\circ} \mathrm{C}$ with $16 \mathrm{~h}$ light and $8 \mathrm{~h}$ dark. RT-PCR was performed after those treatments.

\section{PCR Cloning and Sequence Analysis}

For PCR cloning, a primer CL (PCR cloning $5^{\prime}$ primer) and $\mathrm{CR}$ (PCR cloning 3' primer) were designed based on the conservative base sequences of PGIP genes from Arabidopsis, rape and Chinese cabbage. PCR primer sequences for all experiments are given in Table 1. Total RNA was extracted with an RNeasy plant mini kit (Qiagen, Valencia, CA, USA) from the stigma of blooming flowers of radish and quantified with a spectrophotometer (Beckman Coulter DU 650, Indianapolis, IN, USA). The synthesis of 1 st strand cDNA was completed using a Superscript 1st-Strand Synthesis Kit (Invitrogen, Carlsbad, CA, USA) from $5 \mu \mathrm{g}$ total RNA according to the manufacturer's recommendation. Genomic DNA was extracted from young leaves of radish using a DNeasy Plant Mini Kit (Qiagen) and quantified with a spectrophotometer. The PCR template used was a 1:20 dilution of the 1st strand cDNA product $(1 \mu \mathrm{L})$. PCR was conducted using primer 5' CL and 3' CR. The same primer set was used for the PCR with genomic DNA. PCR amplifications were carried out in a final volume of $20 \mu \mathrm{L}$ containing $10 \mathrm{mM}$ Tris- $\mathrm{HCl}$ (pH 8.4), $1.5 \mathrm{mM} \mathrm{MgCl}_{2}, 50 \mathrm{mM} \mathrm{KCl}, 0.25 \mathrm{mM}$ each dNTP, $0.2 \mu \mathrm{M}$ each primer, $20 \mathrm{ng}$ template DNA and 1 unit of Taq DNA polymerase using the following: $94^{\circ} \mathrm{C}$ for $5 \mathrm{~min}$, then 35 cycles of $\left(94^{\circ} \mathrm{C}\right.$ for $1 \mathrm{~min}, 58^{\circ} \mathrm{C}$ for $1 \mathrm{~min}$, $72^{\circ} \mathrm{C}$ for $1 \mathrm{~min}$ ), with final extension step of $72^{\circ} \mathrm{C}$ for 10 min. PCR products were analyzed on a $1.2 \%$ agarose gel 
Table 1. Primer sequences used for PCR and RT-PCR analyses.

\begin{tabular}{|c|c|c|}
\hline Gene & Primer & Sequence $\left(5^{\prime} \rightarrow 3^{\prime}\right)$ \\
\hline \multirow[t]{2}{*}{ PCR cloning } & CL (PCR cloning 5' primer) & CGCCAAGCTCAAGTATCTCC \\
\hline & CR (PCR cloning 3' primer) & TCGAATTCCTGAAGTGTCCC \\
\hline \multirow[t]{9}{*}{ DNA walking } & 5' TSP1 & AAGCATCGCCTCCGAGCTT \\
\hline & $5^{\prime}$ TSP2 & GTTTTCGGTATATAACCGGAGAGCTGG \\
\hline & $5^{\prime}$ TSP3 & TGGTTGTGTGATAGGTAAAGGTCAGGTCA \\
\hline & $5^{\prime}$ TSP4 & GGGATCTGACCGGTGAGGTTA \\
\hline & 5' TSP5 & GTGAGTTTGCGGAAGACAAGAGTCTGTAG \\
\hline & 5' TSP6 & AAGTCACCGACTTCAGGCGGGAT \\
\hline & 3' TSP1 & GATCACAGGGAATATCCCGGTT \\
\hline & 3' TSP2 & TGGACCGAAGCTCCTCTCCAGTT \\
\hline & 3' TSP3 & TGTGTGGACACATCCCCACAGGA \\
\hline \multirow[t]{2}{*}{$R s P G I P 1$} & RsPGIP-FW & ATAGGGATCCCCATGTGTGGTAAAACAACGACA \\
\hline & RsPGIP-RV & AGTCGTCGACTCACTTGCAACTATCAAGAGGTGC \\
\hline \multirow[t]{2}{*}{ RsACTIN } & RsACTIN-FW & ATGACATGGAGAAGATCTGG \\
\hline & RsACTIN-RV & TGAGCTTGTTTTGGAAGTCT \\
\hline
\end{tabular}

(Agarose M, Biosesang, Seongnam, South Korea) containing $\mathrm{EtBr}$ (Sigma, St. Louis, MO, USA) and the corresponding DNA bands were recovered and cloned into pGEM-T Easy Vectors (Promega, Madison, WI, USA) for sequencing.

DNA sequences of the insert (about $600 \mathrm{bp}$ ) were determined by Genotech Co. Ltd. (South Korea). Sequence analysis was performed using the software BioEdit ver 7.0.5 (www. mbio.ncsu.edu/BioEdit/) and Basic Local Alignment Search Tool (BLAST; Altschul et al., 1990) in the GenBank database (www.ncbi.nlm.nih.gov).

\section{DNA Walking Experiments}

Two walks were performed in the $5^{\prime}$ direction and also in the $3^{\prime}$ direction for a total of three DNA walking experiments using a primer designed based on the confirmed base sequence of RsPGIP. The first experiment in the $5^{\prime}$ direction was carried out by using a DNA walking Speed $U p^{\mathrm{TM}}$ Premix Kit (Seegene, Seoul, South Korea) after designing primers 5' target-specific primer (TSP) 1, 5' TSP2, and 5' TSP3. Since a region that includes the start codon of RsPGIP was not acquired through the first experiment, primers 5' TSP4, 5' TSP5, and 5' TSP6 were designed using the sequence of the acquired $5^{\prime}$ region; the second experiment in the $5^{\prime}$ direction was then performed. Walking the $3^{\prime}$ direction was done using primers 3' TSP1, 3' TSP2, and 3' TSP3, providing sequence data to confirm the gene as RsPGIP.

\section{Sequence Analysis}

The NCBI website was used for the database search and the analysis of the deduced amino acid sequence similarity. A multiple sequence alignment was done with the ClustalW program (www.ebi.ac.uk/clustalw/; Larkin et al., 2007). A phylogenetic tree was generated using the neighbor-joining method by Mega4 (http://www.megasoftware.net/; Kumar et al., 2008). Signal peptide prediction and motif searches were performed using SignalP ver 3.0 (www.cbs.dtu.dk/ services/SignalP/; Bendtsen et al., 2004) and PROSITE (www.expasy.org/prosite/; Hulo et al., 2006), respectively.

\section{Stress Treatments}

To induce wound, leaves were mechanically damaged by crushing the boundary point at the mid-rib with sterile forceps. For cold treatment, 4-week old plants were kept at $4^{\circ} \mathrm{C}$ under $16 \mathrm{~h}$ of daylight for 3 days. For salt treatment, plants were watered, and then their leaves were sprayed with $0.5 \mathrm{M}$ $\mathrm{NaCl}$ every $12 \mathrm{~h}$ for 3 days. For drought treatment, watering was withheld for 4 days, at which time plants began to wilt. For all the treatments, samples were collected from 4-week-old plants at $0,0.5,1,2,4,8,16,24,48$, and $72 \mathrm{~h}$ after treatment. The same age plants treated with Methyl-jasmonic acid (MeJA) were used as positive control, and those without any treatment were used as negative control. After each treatment, leaves were collected and frozen immediately in liquid $\mathrm{N}_{2}$ from three individual plants and stored at $-70^{\circ} \mathrm{C}$ for RNA extraction.

\section{Treatments of Different Defense Response Activators}

The biggest leaves of 4-week-old plants were selected to 
examine the effects of different defense response activators. MeJA (100 $\mu \mathrm{M}$, Sigma, St. Louis, USA) and salicylic acid (50 mM, SA; Sigma, St. Louis, USA) were sprayed on the aerial parts of plants. Plants sprayed with distilled water were used as a control. Leaves were harvested at $0,0.5$, $1,2,4,8,16,24,48$, and $72 \mathrm{~h}$ after treatment.

\section{RNA Purification and RT-PCR Analysis}

For RT-PCR analysis, the total RNA was purified from the frozen sample using the RNeasy Plant Mini Kit (Qiagen) and according to the manufacturer's instructions. cDNA synthesis was performed with the same method of PCR cloning. RT-PCR was carried out using redesigned RsPGIP-FW and RsPGIP-RV primers.

\section{Results}

\section{Isolation of the RsPGIP Gene}

In this study, PCR cloning primers prepared based on the conserved regions of PGIP genes from Arabidopsis, rape, and Chinese cabbage were used to amplify DNA fragments from radish stigma cDNA and genomic DNA. DNA product with an expected size of about $600 \mathrm{bp}$ was amplified by PCR (Fig. 1B). The DNA fragment of $676 \mathrm{bp}$ amplified from genomic DNA was approximately $100 \mathrm{bp}$ longer than
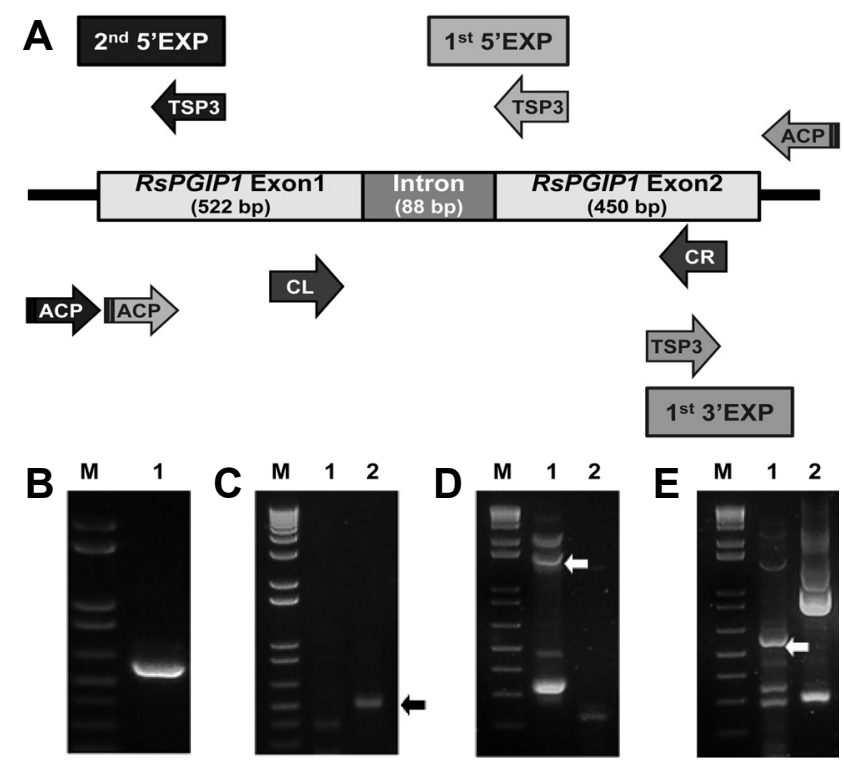

Fig. 1. Gene structure and amplification of the RsPGIP1 gene region. (A) Gene structure of RsPGIP1. CL, PCR cloning 5' primer; $\mathrm{CR}, \mathrm{PCR}$ cloning 3' primer; TSP, target specific primer; $A C P$, annealing controlled primer. (B) PCR cloning product. (C) PCR products of 1 st $5^{\prime}$ region DNA walking experiment, Lane 1: ACP1, Lane 2: ACP2. (D) PCR products of 2nd 5' region DNA walking experiment, Lane 1: ACP1, Lane 2: ACP2. (E) PCR products of 3 ' region DNA walking experiment, Lane 1: ACP1, Lane 2: ACP2. DNA fragments for sequencing are indicated with an arrow. $M$, wide range DNA logical marker (100 bp-10 kb). that amplified from stigma cDNA. Cloning and sequencing of the two products revealed that the $88 \mathrm{bp}$ DNA fragment was an intron of the radish PGIP gene. The intron has good 5' and 3 ' splice site consensus CAG-GT and ACAG-GT respectively (Fig. 1A). High similarities in amino acid sequences were found between radish PGIP cDNA and PGIP from Chinese cabbage, rape, Arabidopsis. The PGIP gene from radish was named $R s P G I P 1$ (Raphanus sativus L. PGIP gene copy No. 1).

The full length ORF sequence of RsPGIPl was confirmed by performing 5' DNA walking twice and 3' DNA walking once. The first 5' DNA walking experiment was performed using primers $5^{\prime} \mathrm{TSP} 1,5^{\prime} \mathrm{TSP} 2$, and 5' TSP3, constructed based on the sequence of partial RsPGIP1 gene and confirmed by PCR cloning. The band with the most distinct 0.5 kilo base $(\mathrm{kb})$ size was confirmed in the lane of DW (DNA Walking) -ACP (Annealing Control Primer) 2. The sequence of this band included the intron ( $88 \mathrm{bp}$ ) area from the location of $5^{\prime}$ TSP3 (587) to 165, and was confirmed as being $510 \mathrm{bp}$ in size (Fig. 1C). Since the translation start site in the DNA walk experiment of the first $5^{\prime}$ DNA walk was not confirmed, primers 5' TSP4, 5' TSP5, and 5' TSP6 were used in the second $5^{\prime}$ DNA walking experiment. They were designed after analysis of the first 5' DNA walk. As a result of performing the second 5 ' DNA walk, clear bands of $0.3,0.6,1.6$, and $2.0 \mathrm{~kb}$ size were detected in the lane of DW-ACP1. Among them, fragments of $0.3 \mathrm{~kb}$ and 1.6 $\mathrm{kb}$ size were cloned in the pGEMT-easy vector and their sequence analyzed. These two bands represented the RsPGIPI gene sequence and the sequence of $1631 \mathrm{bp}$ including the RsPGIP1 promoter region from 5' TSP6 (258) to -1373 (Fig. 1D).

DNA walking from the $3^{\prime}$ direction was performed using primers 3' TSP1, 3' TSP2, and 3' TSP3, constructed based on the DNA sequence of partial RsPGIPl and confirmed with PCR cloning. A variety of clear bands were detected in the DW-ACP1 lane as well as other lanes, but in the DW-ACP1 lane, bands of $5.5 \mathrm{~kb}$ and $1.3 \mathrm{~kb}$ size were detected. Both bands were from the RsPGIPl gene sequence and from the location of 3' TSP6 (868) to 2160, the sequence of 1292 bp was confirmed (Fig. 1E).

Primers RsPGIP-FW and RsPGIP-RV were designed based on the sequence of the full-length ORF of RsPGIP1. cDNA from the RsPGIPl gene was cloned by PCR using the prepared primers and stigma cDNA. The DNA sequence was analyzed by cloning the cDNA of RsPGIP1 into pGEMTeasy. The RsPGIPl gene sequence was confirmed from cDNA and exactly matched the sequence of RsPGIPl confirmed from genomic DNA. The RsPGIPl gene was deposited in GenBank database with accession no. EF392661. 
Structural Analysis of RsPGIP1

The promoter region of $R s P G I P(\mathrm{p} R s P G I P 1)$ was analyzed for the presence of putative $c i s$-acting regulatory elements (Table 2). An element containing the type II MYB consensus sequence (MBSII), a binding site for Myb-related transcription factors, was present at the -588 position. Additional putative cis-acting regulatory elements are present in $\mathrm{p} R$ SPGIP1. A sequence at position -799 showed high similarity to the

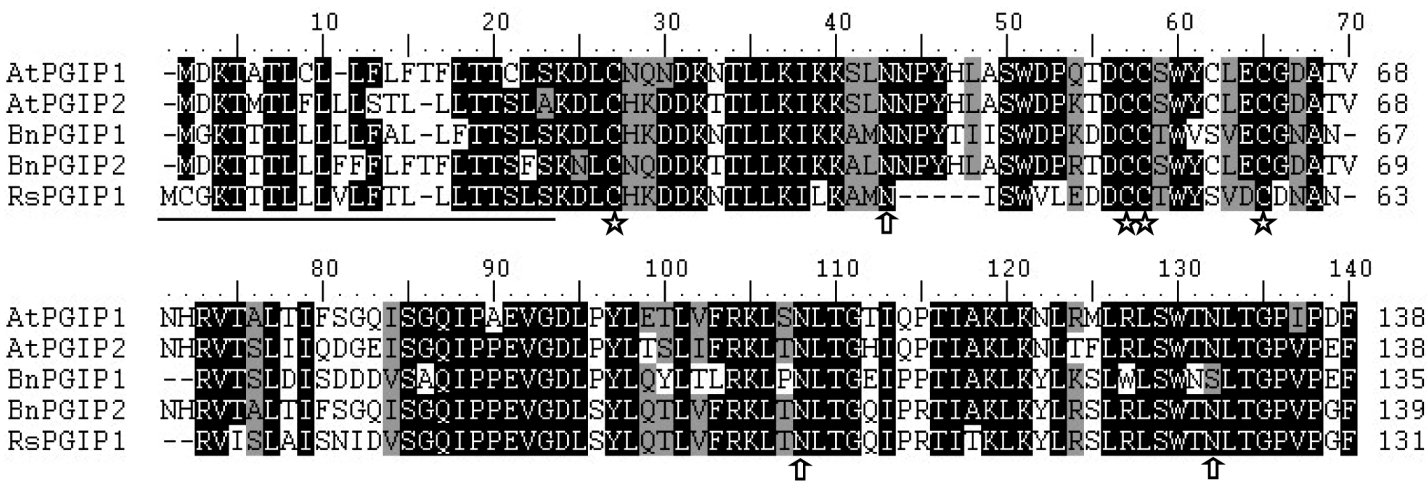

AtPGIP 1

AtPGIP2

BnPGIP1

BnPGIP2

RsPGIP 1

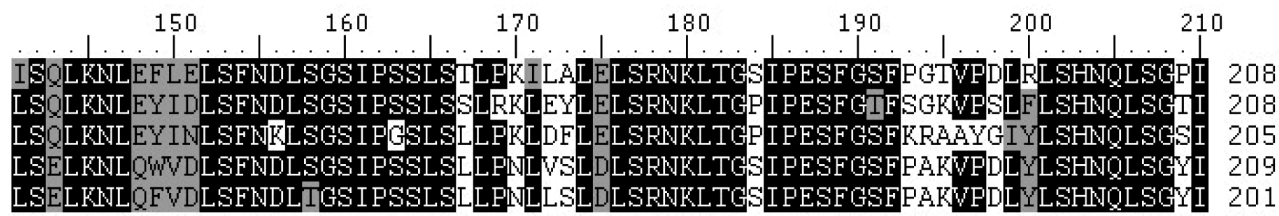

$\lambda$ tPGIP 1

AtPGIP2

BnPGIP1

BnPGIP2

RsPGIP1

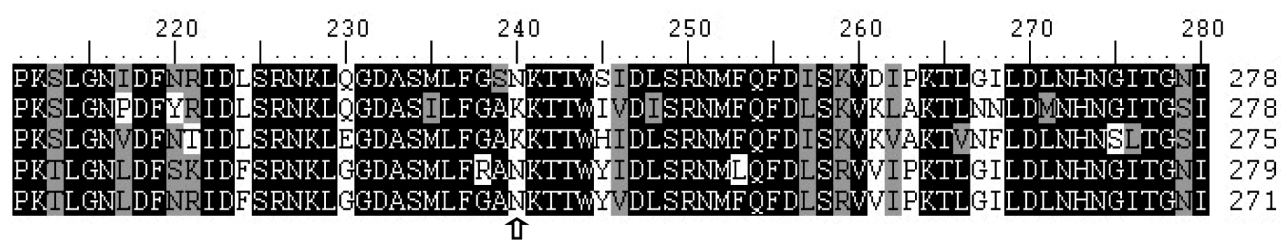

\section{AtPGIP1 \\ AtPGIP2 \\ BnPGIP1 \\ BnPGIP2 \\ $R \leq P G I P 1$}
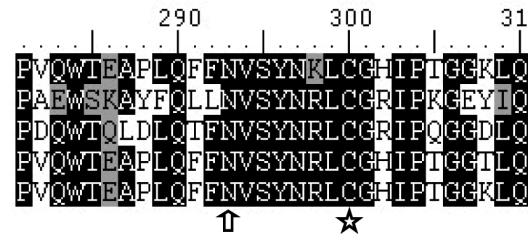

310

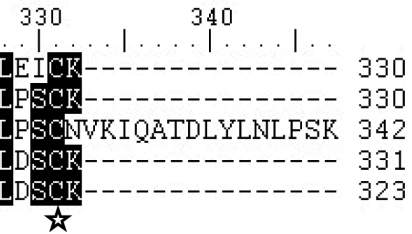

Fig. 2. Alignment of predicted amino acid sequences of PGIPs. The signal peptide of RsPGIP1 is underlined, and the cysteines and glycosylation sites are indicated with a star and upward arrow. The amino acid sequences shown are for AtPGIP1, Arabidopsis thaliana AtPGIP1 (accession no. NM120769); AtPGIP2, Arabidopsis thaliana AtPGIP2 (accession no. NM120770); BnPGIP1, Brassica napus BnPGIP1 (accession no. AF529691); BnPGIP2, Brassica napus BnPGIP2 (accession no. AF529693); RsPGIP1, Raphanus sativus RsPGIP1 (accession no. EF392661).

Table 2. Notable putative cis-acting elements in the RsPGIP1 promoter.

\begin{tabular}{|c|c|c|c|}
\hline \multirow{2}{*}{ Category } & \multirow{2}{*}{ cis Element } & \multicolumn{2}{|c|}{ Rs PGIP1 } \\
\hline & & sequence $^{z}$ & position \\
\hline Myb binding site & MBS II $[a(a / c) c(a / t) a(a / c) c]$ & aaccaac & $-588 /-581$ \\
\hline \multirow[t]{5}{*}{ pathogen response } & LS4 & ttgact & $-444 /-438$ \\
\hline & & & $-324 /-318$ \\
\hline & HSRE & taaaatnttng & $-194 /$ \\
\hline & & & $-186 /$ \\
\hline & & & $-170 /$ \\
\hline Wound induction & PINIIK & aagcgtaagt & -799 \\
\hline
\end{tabular}

${ }^{2}$ Sequence is indicated from the 5 ' to the 3 ' end.

${ }^{y}$ Position of the cis-element with respect to the translation start (5' end/3' end). 
sequence 5'-AAGCGTAAGT-3' found at position -165 of potato proteinase inhibitor II $\mathrm{K}$ promoter that is required for wound-induced expression (Palm et al., 1990). Three putative HSRE elements $(-194,-186,-170)$, which are ATrich motifs conserved in the promoters of several tobacco pathogen-induced genes (Pontier et al., 2001), also are present in $\mathrm{p} R S P G I P 1$. In addition, two sequences identical to the LS4 elements $(-444,-324)$, TTGACT, which acts as a negative regulator of Arabidopsis $P R 1$ expression and as an elicitorresponsive element (W box) in parsley PRl (Ferrari et al., 2003).

Sequence analysis revealed that the ORF of RSPGIP1 is 972 bp in length. Domain analysis revealed that the fulllength $R s P G I P 1$ gene contains an ORF encoding a protein of 323 amino acids and has a conserved $N$-terminal sequence motif and an LRR region (Figs. 2 and 3 ). The deduced amino acid sequence of the ORF of RsPGIPl has a high degree of similarity with previously isolated PGIPs and contains features characteristic of PGIPs found in other plants. The ORF of RsPGIP1 gene encodes for a polypeptide that shows 86, 84, 75, 63, 63, 61, 61, 61, 59, 58, 56, 56, 56, and $43 \%$ similarity with PGIPs from Chinese cabbage, rape, Arabidopsis, apricot, peach, pear, apple, cotton, citrus, grape, potato, tomato, and bean, respectively. Fig. 4 shows the phylogenetic relationship of amino acid sequences between radish RsPGIP1 and other PGIPs. The encoded polypeptide has common features of other PGIP proteins. The ORF of RsPGIP1 showed 88.1, 84.0 and 76.3\% similarity with PGIPs from Chinese cabbage (BrPGIP2, NCBI accession no. FJ771027), rape (BnPGIP2, NCBI accession no. AF529693), and Arabidopsis (AtPGIP1, NCBI accession no. NM120769), respectively.

The predicted molecular weight of the RsPGIP1 deduced from the amino acid sequences is $36 \mathrm{kDa}$ and the isoelectric point 8.31 [Peptide Mass]. The RsPGIP1 gene product has high leucine content (54 of 323 aa) including 39 aa that are highly conserved. The region spanning nearly $74 \%$ of

\begin{tabular}{|c|c|c|c|}
\hline RVIS - & LAISN- & IDVSGQIPPEVGDLS & 1 \\
\hline YLQT - & LVFRKL & TNLTGQIPRTITKLK & 2 \\
\hline YLRS - & LRLSW- & TNLTGPVPGFLSELK & 3 \\
\hline NLQF - & VDLSF- & NDLTGSIPSSLSLLP & 4 \\
\hline NLLS - & LDLSR- & NKLTGSIPESFGSFP & 5 \\
\hline AKVPD & LYLSH- & NQLSGYIPKTLGNLD & 6 \\
\hline F NR - - & IDFSR- & NKLGGDASM-LFGAN & 7 \\
\hline KTTWY & VDLSR- & NMF-QFDLSRVVIPK & 8 \\
\hline T L G I - & LDLNH- & NGITGNIPVQWTEA & 9 \\
\hline PLQF - & LNVSY- & NRLCGHIPTG-GKLQ & 10 \\
\hline
\end{tabular}

Fig. 3. Ten putative leucine-rich repeats (LRRs) in RsPGIP1. Consensus: $x$, any amino acid; $L$, leucine or aliphatic amino acid; I, isoleucine; $\mathrm{T}$, serine/threonine; $d$, acidic residue. the mature peptide (from amino acid 64 to 301) contains ten conserved LRR motifs (xLxxLdLSxNxLTGxIPxxLxxLx) with an average size of 24 aa (Fig. 3), where ' $L$ ' indicates leucine residue or any aliphatic amino acid residue, ' $d$ ' did the acidic amino acid residue and ' $x$ ' can be any amino acid. The LRR domain, which has a conserved region, can form a $\beta$-sheet/ $\beta$-turn motif, assumed to be an Avirulence (Avr) protein recognition site that is an important part of common cloned plant R genes (Di Matteo et al., 2006; Federici et al., 2006). LRR domains are suggested to be involved in defense signal activation by interacting with a signaling component or binding to a ligand (Worrall et al., 1998).

Analysis using the ExPASy proteomics server of the Swiss Institute of Bioinformatics (SIB) revealed that radish RsPGIP1 has five potential $N$-glycosylation sites $(\mathrm{NXS} / \mathrm{T})$ found in conserved positions and also a number of potential phosphorylation sites including the presence of eight highly conserved cysteine residues, four in the first 65 aa and four in the final 32 aa of the mature peptide. Based on SignalP-HMM (Hidden Markov Models) and SignalP- $N, N$ (neural networks) prediction to $N$-terminal peptides, as determined by amino acid sequencing, radish RsPGIP1 has an $N$-terminal signal peptide of 22 amino acids (Fig. 2). The putative cleavage site is between

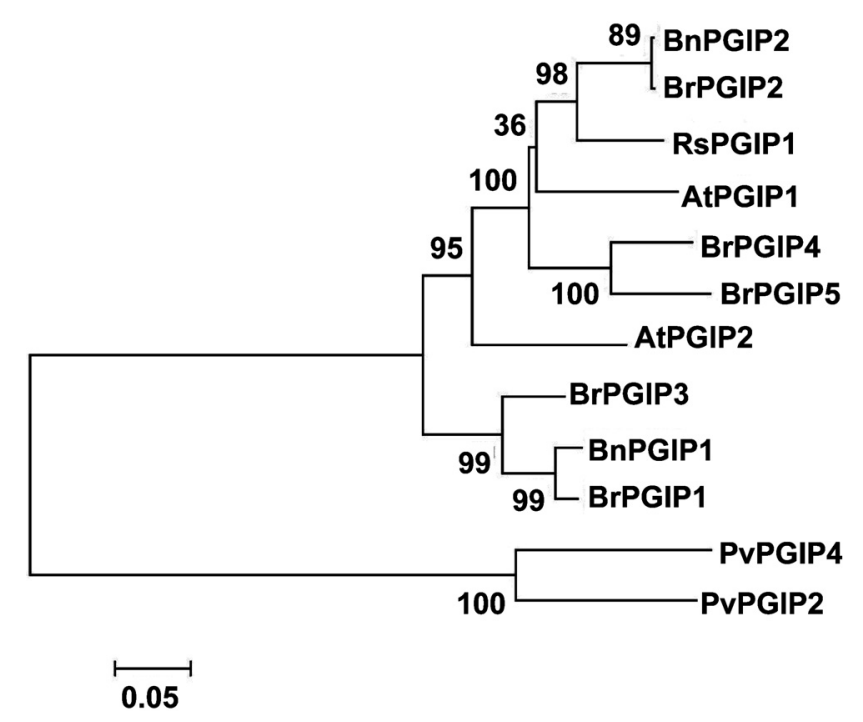

Fig. 4. Phylogenetic tree of RsPGIP1 and the other PGIPs. The tree was constructed from amino acid sequences by the neighbor-joining method of ClustalW and Mega4 (http://www. megasoftware.net/). Numbers at the nodes are bootstrap values from 100 replications and represent weighted amino acid substitutions, with the scale bar represents $10 \%$ weighted sequence divergence. At, Arabidopsis thaliana; Br, Brassica rapa; Pv, Phaseolus vulgaris. AtPGIP1 (accession no. NM120769); AtPGIP2 (accession no. NM120770); BrPGIP1 (accession no. FJ898288); BrPGIP2 (accession no. FJ771027); BrPGIP3 (accession no. FJ898286); BrPGIP4 (accession no. FJ898289); BrPGIP5 (accession no. FJ898287); PVPGIP2 (accession no. FM253103); PVPGIP4 (accession no. AJ786411). 
the 22nd and 23rd aa (SLS-KD) as shown with an underline, and the 22nd aa was confirmed to be serine, also well conserved in other PGIPs.

\section{Organ Specificity in RsPGIP1 Expression}

We examined tissue-specific expression of RsPGIP1. The expression of RsPGIPl was observed in three organs of Raphanus sativus, roots, leaves, and stigma. However there was a quantitative difference in terms of expression level. Less expression was observed in the leaves than in stigma or primary root from the flowering plants (Fig. 5). Also, the enrichment of $R s P G I P 1$ transcripts in stigma showed similar pattern as the case of BrPGIPs in Chinese cabbage (Hwang et al., 2010).

Effect of Different Stresses on the Transcript Levels of the RsPGIP1 Gene

When Raphanus sativus plants were treated with different

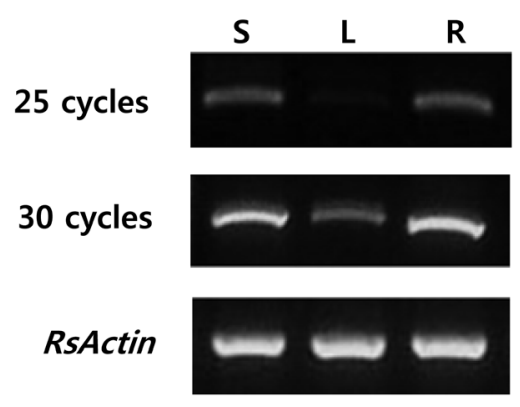

Fig. 5. RT-PCR analysis of RsPGIP1 gene expression in plant organs. S, stigma; L, leaf blade; R, primary root. Total RNA was extracted from tissues of radish plants when in full-bloom.

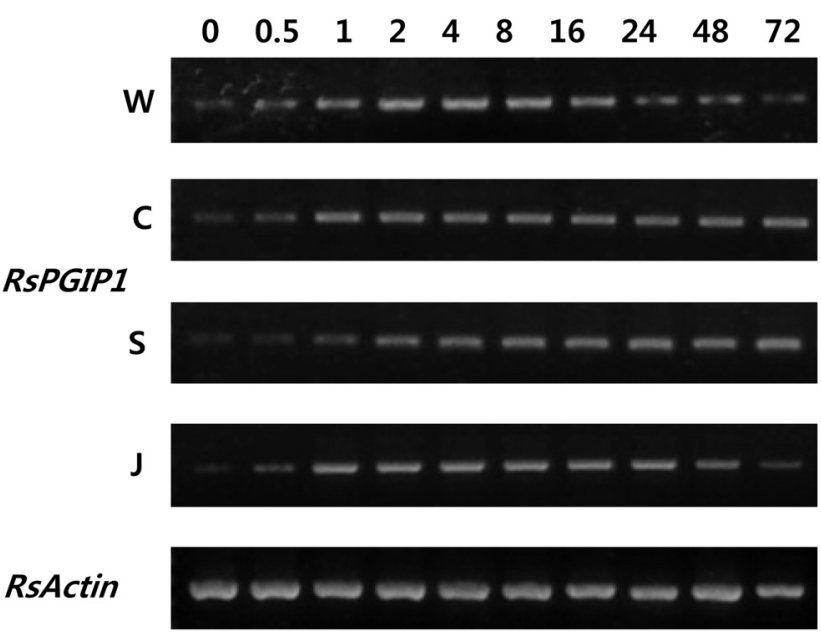

Fig. 6. Effect of mechanical wounding, low temperature, salt, and jasmonic acid treatments on the RsPGIP1 gene induction. RT-PCR reaction with primers for actin was performed to determine quantitative equality of the RNA templates used for samples. W, mechanical wounding; C, low temperature; $\mathrm{S}$, salt; J, jasmonic acid. environmental stresses such as mechanical wounding, cold temperature, high salt, and dehydration (Fig. 6), RT-PCR revealed that the $R S P G I P 1$ gene was induced rapidly in response to mechanical wounding, in which a peak was shown 2 to $4 \mathrm{~h}$ after treatment. Also, the gene was induced in plants treated with low temperature and salt. Transcript levels of RSPGIP1 increased gradually between 1 and 72 $\mathrm{h}$ after salt treatment, whereas, in the case of dehydration, induction levels did not change (Data was not shown). In all the stress treatments, accumulation of transcripts was low, and bands were not detected after 30 cycles of RT-PCR. However, after 35 cycles induction was confirmed. In all experiments, the 4-week-old leaves without any treatment were used as a control. In the control, transcript of RsPGIPI was low and was only detected by RT-PCR after 35 cycles.

We observed tissue-specific expression of RsPGIPl at the flowering stage (Fig. 5). Interestingly, transcript level of $R s P G I P 1$ in the leaves from the flowering plants was higher than 4-week-old leaves (Figs. 5 and 6). This suggests that the expression of $R s P G I P 1$ is differentially regulated by developmental stage.

Activities of Different Defense Response Activators in the RsPGIP Gene Expression

When treated with MeJA and SA, only MeJA induced the RsPGIPl gene in leaves of 4-week-old $R$. sativus in response to defense response activators. Expression was rapidly induced after MeJA treatment at 1, 2, and $4 \mathrm{~h}$, and then steadily decreased. Transcription was highest from 1 to $4 \mathrm{~h}$ after treatment (Fig. 6).

\section{Discussion}

Cloned a Raphanus sativus L. PGIP gene using PCR cloning and DNA walking was named as RsPGIP1. The gene has similar domain structures and sequence homology to other PGIPs, including the Brassicaceae family; the highest match in deduced amino acid sequence among them was $86 \%$.

PGIPs are reported to be induced in many plant tissues under various environmental conditions and have been found in many plant species. In our study, the constitutive expression of the RsPGIPl was confirmed in the stigma. To a lesser extent, accumulation of transcripts was also observed in leaves of flowering radish plants (Fig. 5). Conversely, leaves from 4-week-old plants showed relatively low expression of $R s P G I P 1$ in the absence of applied stress or any defense response activator treatments (Fig. 6). These results are similar to the expression pattern of the BnPGIPs (Li et al., 2003) and BcPGIP (Ahsan et al., 2005). The expression of RsPGIP1 
is also observed by mechanical wounding as reported in PGIPs from Arabidopsis (Li et al., 2003), Chinese cabbage (Ahsan et al., 2005), strawberry (Mehli et al., 2004), and bean (Devoto et al., 1998). Mechanical wounding is generally generated in the infection site where defense-related genes may be active against pathogens (Schenk et al., 2003).

The BrPGIP2 gene, characterized from Chinese cabbage provides us with the clue on the molecular mechanism on disease resistance. A study of the inoculation of Pcc, a bacterial pathogen causing soft rot disease, revealed that the expression of BrPGIP2 from J3-11M-54, the relatively resistant Chinese cabbage line was strongly and rapidly induced compared to J3-11M-3, a relatively susceptible line. Further analysis of transgenic plants, obtained by the overexpression in tobacco and Chinese cabbage with BrPGIP2 proved that resistance against soft rot disease of transgenic plants increased by more than $50 \%$, being ascribed to the promotion of BrPGIP2 activity (Hwang et al., 2010).

It is recognized that PGIPs play an important role in plant defense through studies on improvement of disease resistance involving over-expression of PGIP genes and elucidation of the expression pattern after treatments with several microbial defense elicitors (Di Matteo et al., 2006; Federici et al., 2006; Ferrari et al., 2006). It is known that PGIPs inhibit the action of pathogen $\mathrm{PG}$ on cell wall pectic structural components in various plants. This inhibition was investigated through site-directed mutagenesis of particular amino acid residues in the active site and structural portions of PG protein (Federici et al., 2001; Manfredini et al., 2006; Spadoni et al., 2006; van Santen et al., 1999).

Interestingly, RsPGIPl was induced by environmental stress such as low temperature and salt (Fig. 6). Low temperature induction of PGIP expression has been reported in Brassica napus (Wao et al., 1999), Arabidopsis (Ferrari et al., 2003), Chinese cabbage (Ahsan et al., 2005), and apple (Yao et al., 1999). Induction of PGIP by salt stress has been reported in Chinese cabbage (Ahsan et al., 2005). With Arabidopsis PGIP genes, expression was not induced by drought or heat stress (Rizhsky et al., 2003). Likewise, in the case of the RsPGIP1 gene, there was no response to drought stress. In the promoter region of $R s P G I P 1$, there exists a PINIIK (ciselement) that is related to wounding. Also, LS4 and HSRE exist in relation to pathogen response (Table 2). Therefore, it is assumed that each gene responds selectively to different environmental stress. We observed that RsPGIPl gene was induced by JA (Fig. 6). Jasmonic acid is the end product in the octadecanoid pathway involving sequential action of allene oxide synthase (AOS), allene oxide cyclase (AOC) and 12-oxo-PDA-reductase (OPR). This pathway mediates plant response to a wide range of biotic and environmental stress (Agrawal et al., 2003). A variety of environmental cues can induce the expression of AOS, AOC, and OPR genes in plants and therefore production of relevant enzymes. It has been reported that the rice $O P R 1$ gene is upregulated by many environmental factors, such as: wounding, heavy metals, osmotic pressure, UV-C irradiation, fungal elicitors, protein phosphatase inhibitors and gaseous pollutants (Agrawal et al., 2003). Since the RsPGIPl gene was induced by mechanical wounding, various environmental stress, and JA, we conclude that the JA signal transduction pathway may regulate $R S P G I P 1$ gene expression. It has also been demonstrated in other systems that induction of defense-related genes by mechanical wounding and JA involves the JA-dependent signal transduction pathway (Pieterse and van Loon, 1999). Based on the above, we postulate that RsPGIPl gene expression is also regulated by the JA signal transduction pathway, but more research regarding the relationship between JA and other environmental factors is needed.

The RsPGIPl gene isolated from radish 'Daejinyeorum' will be useful in research on the molecular mechanism of the resistance to diseases in radish. More investigations are required to extend our knowledge on understanding the mechanism by which RsPGIP1 plays a decisive role in postharvest diseases like soft rot in radish as well as other Brassicaceae crops.

\section{Literature Cited}

Agrawal, G.K., N.S. Jwa, J. Shibato, O. Han, H. Iwahashi, and R. Rakwal. 2003. Diverse environmental cues transiently regulate OsOPR1 of the "octadecanoid pathway" revealing its importance in rice defense/stress and development. Biochem. Biophys. Res. Commun. 310:1073-1082.

Ahsan, N., H.S. Yoon, and J. Jo. 2005. Molecular cloning of a BcPGIP cDNA from Brassica campestris and its expression to several stresses. Plant Sci. 169:1081-1089.

Bendtsen, J.D. H. Nielsen, G. von Heijne, and S. Brunak. 2004. Improved prediction of signal peptides: SignalP 3.0. J. Mol. Biol. 340:783-795

Cheigh, H. S. and K.Y. Park. 1994. Biochemical, microbiological, and nutritional aspects of kimchi (Korean fermented vegetable products). Crit. Rev. Food Sci. Nutr. 34:175-203.

De Lorenzo, G., R. D’Ovidio, and F. Cervone. 2001. The role of PG-inhibiting proteins (PGIPS) in defense against pathogenic fungi. Annu. Rev. Phytopathol. 39:313-335.

Devoto, A., F. Leckie, E. Lupotto, F. Cervone, and G. De Lorenzo. 1998. The promoter of a gene encoding a PG-inhibiting protein of Phaseolus vulgaris L. is activated by wounding but not by elicitors or pathogen infection. Planta 205:165-174.

Di Matteo, A., D. Bonivento, D. Tsernoglou, L. Federici, and F. Cervone. 2006. PG-inhibiting protein (PGIP) in plant defense: A structural view. Phytochemistry 67:528-533.

Federici, L., C. Caprari, B. Mattei, C. Savino, A. Di Matteo, G. 
De Lorenzo, F. Cervone, and D. Tsernoglou. 2001. Structural requirements of endoPG for the interaction with PGIP (PGinhibiting protein). Proc. Natl. Acad. Sci. 98:13425-13430.

Federici, L., A. Di Matteo, J. Fernandez-Recio, D. Tsernoglou, and F. Cervone. 2006. PG inhibiting proteins: Players in plant innate immunity? Trends Plant Sci. 11:65-70.

Ferrari, S., D. Vairo, F.M. Ausubel, F. Cervone, and G. De Lorenzo. 2003. Tandemly duplicated Arabidopsis genes that encode PG-inhibiting proteins are regulated coordinately by different signal transduction pathways in response to fungal infection. Plant Cell 15:93-106.

Ferrari, S., R. Galletti, D. Vairo, F. Cervone, and G. De Lorenzo. 2006. Antisense expression of the Arabidopsis thaliana AtPGIP1 gene reduces PG-inhibiting protein accumulation and enhances susceptibility to Botrytis cinerea. Mol. Plant-Microbe Interact. 19:931-936.

Fuchigami, M. 1987. Relationship between maceration and pectic compositions and the softening of the texture of Japanese radish roots during cooking. J. Food Sci. 52:1317-1320.

Gregori, R., M. Mari, P. Bertolini, J.A. Sanudo Barajas, J.B. Tian, and J.M. Labavitch. 2008. Reduction of Colletotrichum acutatum infection by a polygalacturonase inhibitor protein extracted from apple. Postharvest Biol. Technol. 48:309-313.

Hulo, N., A. Bairoch, V. Bulliard, L. Cerutti, E. De Castro, P.S. Langendijk-Genevaux, M. Pagni, and C.J.A. Sigrist. 2006. The PROSITE database. Nucleic Acids Res. 34:D227-D230.

Hwang, B-H., H. Bae, H.-S. Lim, K.B. Kim, S.J., Kim, M.-H., Im, B.-S. Park, D.S. Kim, and J. Kim. 2010. Overexpression of polygalacturonase-inhibiting protein 2 (PGIP2) of Chinese cabbage (Brassica rapa ssp. pekinenesis) increased resistance to the bacterial pathogen Pectobacterium catovorum ssp. carotovorum. Plant Cell Tiss. Organ Cult. 103:293-305.

Jang, S., B. Lee, C. Kim, S.J. Kim, J. Yim, J.J. Han, S. Lee, S.R. Kim, and G. An. 2003. The OsFOR1 gene encodes a PG-inhibiting protein (PGIP) that regulates floral organ number in rice. Plant Mol Biol. 53:357-372.

Juge, N. 2006. Plant protein inhibitors of cell wall degrading enzymes. Trends Plant Sci. 11:359-367.

Kumar, S., J. Dudley, M. Nei, and K. Tamura. 2008. MEGA: A biologist-centric software for evolutionary analysis of DNA and protein sequences. Briefings Bioinformatics 9:299-306.

Larkin, M.A., G. Blackshields, N.P. Brown, R. Chenna, P.A. McGettigan, H. McWilliam, F. Valentin, I.M. Wallace, A. Wilm, R. Lopez, J.D. Thompson, T.J. Gibson. and D.G. Higgins. 2007. ClustalW and ClustalX version 2. Bioinformatics 23: 2947-2948.

Lebeda, A., D.A.C. Pink, and B. Mieslerovao. 2001. Host-parasite specificity and defense variability in the Lactuca spp. Bremia lactucae pathosystem. J. Plant Pathol. 83:25-35.

Liang, F., K. Zhang, C. Zhou, F. Kong, J. Li, and B. Wang. 2005. Cloning, characterization and expression of the gene encoding PG-inhibiting proteins (PGIPs) of peach [Prunus persica (L.) Batch]. Plant Sci. 168:481-486.

Li, R., R. Rimmer, M. Yu, A.G. Sharpe, G. Seguin-Swartz, D. Lydiate, and D.D. Hegedus. 2003. Two Brassica napus PG inhibitory protein genes are expressed at different levels in response to biotic and abiotic stresses. Planta 217:299-308.

Manfredini, C., F. Sicilia, S. Ferrari, D. Pontiggia, G. Salvi, C. Caprari, M. Lorito, and G.D. Lorenzo. 2006. PG-inhibiting protein 2 of Phaseolus vulgaris inhibits BcPG1, a PG of Botrytis cinerea important for pathogenicity, and protects transgenic plants from infection. Physiol. Mol. Plant Pathol. 67:108-115.

Mehli, L., J.G. Schaart, T.D. Kjellsen, D.H. Tran, E.M.J. Salentijn, H.J. Schouten, and T.H. Iversen. 2004. A gene encoding a PG-inhibiting protein (PGIP) shows developmental regulation and pathogen-induced expression in strawberry. New Phytol. 163:99-110.

Palm, C.J., M.A. Costa, G. An, and C.A. Ryan. 1990. Woundinducible nuclear protein binds DNA fragments that regulate a proteinase inhibitor II gene from potato. Proc. Natl. Acad. Sci. 87:603-607.

Pieterse, C.M.J. and L.C. van Loon. 1999. Salicylic acid-independent plant defense pathways. Trends Plant Sci. 4:52-58.

Pontier, D., C. Balagueo, I. Bezombes-Marion, M. Tronchet, L. Deslandes, and D. Roby. 2001. Identification of a novel pathogen-responsive element in the promoter of the tobacco gene HSR203J, a molecular marker of the hypersensitive response. Plant J. 26:495-507.

Rizhsky, L., H. Liang, and R. Mittler. 2003. The water-water cycle is essential for chloroplast protection in the absence of stress. J. Biol. Chem. 278:38921-38925.

Schenk, P.M., K. Kazan, J.M. Manners, J.P. Anderson, R.S. Simpson, I.W. Wilson, S.C. Somerville, and D.J. Maclean. 2003. Systemic gene expression in Arabidopsis during an incompatible interaction with Alternaria brassicicola. Plant Physiol. 132:999-1010.

Spadoni, S., O. Zabotina, A. Di Matteo, J.D. Mikkelsen, F. Cervone, G. De Lorenzo, B. Mattei, and D. Bellincampi. 2006. PG-inhibiting protein interacts with pectin through a binding site formed by four clustered residues of arginine and lysine. Plant Physiol. 141:557-564.

Van Santen, Y., J.A.E. Benen, K.H. Schroter, K.H. Kalk, S. Armand, J. Visser, and B.W. Dijkstra. 1999. 1.68-A crystal structure of endo-PG II from Aspergillus niger and identification of active site residues by site-directed mutagenesis. J. Biol. Chem. 274:30474-30480.

Wang, C.Y., 1998. Methyl jasmonate inhibits postharvest sprouting and improves storage quality of radishes. Postharvest Biol. Technol. 14:179-183.

Worrall, D., L. Elias, D. Ashford, M. Smallwood, C. Sidebottom, P. Lillford, J. Telford, C. Holt, and D. Bowles. 1998. A carrot leucine-rich-repeat protein that inhibits ice recrystallization. Science 282:115-117.

Yao, C., W.S. Conway, R. Ren, D. Smith, G.S. Ross, and C.E. Sams. 1999. Gene encoding PG inhibitor in apple fruit is developmentally regulated and activated by wounding and fungal infection. Plant Mol. Biol. 39:1231-1241.

York, W.S, A.G. Darvill, M. McNeil, T.T. Stevenson, and P. Albersheim. 1985. Isolation and characterization of plant cell walls and cell wall components. Methods Enzymol. 118:3-40. 